Research Article

\title{
Carbon Nanomaterial Manufacturing System and Automatic Synthesis Equipment and Its Control Device and Control Methods
}

\author{
Gaoxiang Lou $\mathbb{D}$ and Zongyan Cai \\ School of Construction Machinery, Chang'an University, Xi'an, Shannxi 710064, China \\ Correspondence should be addressed to Gaoxiang Lou; 2015025007@chd.edu.cn
}

Received 6 August 2020; Revised 6 September 2020; Accepted 20 October 2020; Published 3 November 2020

Academic Editor: Tifeng Jiao

Copyright (c) 2020 Gaoxiang Lou and Zongyan Cai. This is an open access article distributed under the Creative Commons Attribution License, which permits unrestricted use, distribution, and reproduction in any medium, provided the original work is properly cited.

\begin{abstract}
In recent years, people are committed to developing new technologies and technologies for energy storage and conversion, environmental detection, high-performance sensors and energy security, and other aspects of the increasingly prominent problems in the field of environmental and biosafety. The purpose of this paper is to explore the manufacturing system and automatic synthesis equipment of carbon nanomaterials, understand the control device and control method, and analyze the structure and morphology characteristics of three kinds of carbon nanomaterials produced by carbon nanomaterial manufacturing system by X-ray diffraction and infrared spectroscopy. The results show that the carbon nanomaterial manufacturing system and automatic synthesis system in this paper solve the problems of high cost, low efficiency, and small scale of the existing carbon nanomaterials manufacturing and achieve the precision control of automatic production, so that the productivity is increased by $20 \%-35 \%$, and the cost is reduced by $15 \%-30 \%$. Therefore, they are widely used in the fields of science and technology, environmental protection, and intelligent manufacturing broad prospects. Carbon nanotube manufacturing equipment and automatic synthesis equipment have great production advantages, which can greatly improve the quality and efficiency of carbon nanomaterials. UPY, GO, and UGO carbon nanomaterials produced by carbon nanotube manufacturing equipment are not easy to fall off from the materials. When the wavelength is $500 \mathrm{~nm}$, the absorption frequency of the three materials is the largest. With the extension of the spectral wavelength, the absorption frequency of the three materials is reduced by $52 \%, 33 \%$, and $34.7 \%$, respectively.
\end{abstract}

\section{Introduction}

In recent years, due to the increasingly prominent problems in the field of energy security, environment, and biosecurity, people are committed to solving these severe challenges and research and development for energy storage and conversion, environmental detection, performance sensors, and other aspects. Because the size of the nanomaterial is within $100 \mathrm{~nm}$, it has a high specific surface area and a special morphological structure, so it has unique physical and chemical properties. With the increasing demand for highperformance devices and efficient technologies, people are paying more and more attention to nanomaterials with advanced functions. Advances in nanotechnology and nanomaterials provide new directions for the design and development of electronic devices, sensors, lithium-ion batteries, environmental testing, and other fields.

The research and development of carbon nanomaterial manufacturing systems have greatly promoted the synthesis of carbon nanomaterials and enhanced the application of carbon nanomaterials in the fields of science, technology, and environment. Fernandes modified the glassy carbon electrode with carbon nanocomposites in his research. Carbon nanocomposites show high stability and have a high current intensity and easy to distinguish REDOX peak [1]. Agata introduced research results related to transparent heating elements made of carbon nanomaterials and adopted an alternative method of vacuum deposition of transparent resistance layers and etching low resistance patterns, aiming to make carbon nanomaterials easy to 
realize in large-scale applications [2]. In the experiment, Lee demonstrated the method of using a solution plasma system to produce low-dimensional carbon nanomaterials and its application in flexible conductive paper and compared and studied the effects of graphite rod diameter and ferritin molecular concentration on the production of carbon nanomaterials, and it is verified that carbon nanotubes can be synthesized in this way [3]. Izumi studied the carbon black material in arc black manufactured by chemical vapor deposition (ACB) and carbon nanocoil (CNC) through arc discharge and compared activated carbon (AC) with carbon nanomaterials of various specific capacitances. The reason for using carbon nanomaterials to maintain specific capacitance even at high scan rates is almost the same [4]. Zhang pointed out in his research that carbon nanomaterials have made great progress. Carbon nanomaterials are a better choice for manufacturing biosensors, where higher electron mobility, high chemical stability, electrochemical activity, and higher electric points can promote enzyme adsorption, biocompatibility, and piezoelectric properties, introduce the latest trends in carbon nanotube synthesis, analyze the performance of biosensors, and summarize future challenges and prospects [5]. In summary, the research and development of carbon nanomaterial manufacturing systems have a very broad application prospect.

Automated synthesis equipment is an advanced industrial technology that can greatly improve people's labor productivity. There are many types of research on automated synthesis equipment. In his research, Alur explored the performance advantages of automated synthesis equipment, gave automated synthesis equipment a specification and a set of candidate programs, and successfully found a candidate program that meets the specifications, indicating that automated synthesis equipment already has certain intelligence [6]. Khan pointed out in the article that automated synthesis equipment is a favorable choice for future quantum computing technology because they have multiple advantages over existing nonautomated equipment. You can easily combine classic binary functions by combining two bits into a quaternion value. Expressed as a quaternary function, it is widely used in the scientific field [7]. Osipov considered the problem of automatic synthesis equipment action programs in a constantly changing environment, studied the deductive and comprehensive capabilities of automated synthesis equipment cycles and self-replicating programs and proposed a method for synthesizing such action programs under various possible conditions [8]. Bazhenov introduced a new nuanced analysis of the chemical conversion that occurs during the synthesis of guanidine phosphate oligonucleotides (PGOs) by automated synthesis equipment, which gives the automated synthesis equipment better stability under deblocking conditions [9]. Satsangi confirmed in his research that the design of the reversible system of automated equipment is very different from the traditional system, explored evolutionary algorithms, and used enhanced quantum heuristic evolutionary algorithms to synthesize digital circuits and reference circuits of various automated synthesis equipment, and evolved with other algorithms, and existing search and optimization techniques were compared for performance analysis [10]. It can be seen that the application fields of automated synthesis equipment are extensive, which is of great help to human production and life.

This article discusses the control device and control method of the carbon nanomaterial manufacturing system and automatic synthesis equipment in detail. The research results show that carbon nanotube manufacturing equipment and automated synthesis equipment have great production advantages, which can greatly improve the quality and efficiency of carbon nanomaterials. The carbon nanomaterial manufacturing system and automated synthesis system studied in this paper solve the existing carbon nanomaterials. The problems of high manufacturing cost, low efficiency, and small scale and the precision control of automated production have been achieved, which has increased productivity by $20 \%-35 \%$ and cost by $15 \%-30 \%$. Therefore, they have potential in science and technology, environmental protection, and intelligence. Manufacturing and other fields have broad prospects. The innovation of this article is that by analyzing and comparing the three materials produced by the carbon nanomaterial manufacturing system, it is found that compared with the traditional production method, the carbon nanomaterial manufacturing system has certain stability and reliability and is a carbon nanomaterial application in the field of environmental protection, and scientific research provides new ideas.

\section{Materials and Methods}

This experiment studied the working principle of the carbon nanomaterial manufacturing system and automatic synthesis equipment, as well as the advantages and disadvantages of its control device and control equipment. The carbon nanomaterials produced by the carbon nanomaterial manufacturing system are divided into three types of samples: UPY, GO, and UGO, and were compared with the carbon nanomaterials produced by the traditional carbon nanomaterial manufacturing method to compare the advantages of the carbon nanomaterial manufacturing system. In addition, this study also introduces a set of the intelligent control system for high-throughput nanomaterial synthesis and mainly introduces the control device of automatic synthesis equipment and the control method of each reaction, equipped with an electromagnetic stirrer and electric heating module, which are independent of rotational speed, temperature control, and six bidirectional monomer feeding peristaltic pumps. The Cartesian robot is responsible for the reagent injection, accurate movement to the reaction, the reaction of stirring speed, temperature, and the amount of each reagent which is controlled by the computer and realtime detection [11]. Automated synthesis equipment can be used to synthesize high-throughput materials such as carbon nanomaterials by coprecipitation and hydrothermal methods [12].

The main chemical reagents and raw materials are as follows: sublimation sulfur, ethyl tetra silicate, acetyl trimethyl ammonium chloride, ethanolamine, 1-oleic acid, CAI alkane of ammonium nitrate, sucrose, hydrofluoric 
acid, absolute ethanol, sulfurization carbon, multiwalled carbon nanotubes (diameter 20 to $50 \mathrm{~nm}$ ), multiwalled carbon nanotubes (diameter 60-100 nm), ammonia, sodium hydroxide, piano black, nitric acid, potassium stannate, nitrate hexahydrate. Zinc nitrate hexahydrate, methanol, urea, $\mathrm{N}$-methyl alkenone, 2-1 imidazole bis(II) fluorinated methylamine imide lithium, 1,2 dioxane, and 1,3 dioxolane and lithium nitrate.

The main experimental instruments and equipment are as follows: desktop X-ray diffractometer (Rigaku Corporation), field emission scanning electron microscope (Hitachi), field emission transmission electron microscope (US FEI Corporation), ASAP2020 automatic specific surface area and porosity analyzer (Micromeritic Corporation), TA449F3 thermogravimetric Analyzer (Netsch), Ultrasonic Cleaning Machine (Kunshan Ultrasonic Instrument Co., Ltd.), Glove Box (Mirounga (China) Co., Ltd.), High-Speed Refrigerated Centrifuge (Shanghai Tianshan Instrument Equipment Engineering Co., Ltd.), Electric Thermostat Oil bath pot (Zhengzhou Great Wall Industry and Trade Co., Ltd.), charge and discharge tester (Jinmao Electronics Co., Ltd.), etc. Other instruments and equipment used in this experiment are shown in Table 1.

\section{Carbon Nanomaterial Chemical Property Test}

After the manufacture of the carbon nanomaterial manufacturing system is completed, the carbon nanomaterials prepared by the system are tested. First, the elemental composition and structure of the material were analyzed by X-ray diffraction. The test voltage was $30 \mathrm{kV}$, the current was $15 \mathrm{~mA}$, and the X-ray wavelength during the experiment was set to $0.15418 \mathrm{~nm}$. Next, the surface morphology of the material sample is checked by scanning electron microscope (SEM), and the qualitative and semiquantitative inspection of the sample is checked by X-ray energy dispersive spectroscopy (EDS) attached to it [13]. The preparation of the test sample was as follows: paste the sample on the sample stage using conductive glue (if the sample has poor conductivity, it needs to be sprayed with gold), and then put the sample into the sample chamber of the scanning electron microscope and evacuate it with a vacuum instrument. Observe the morphology of the sample and test its composition [14].

Carbon nanomaterials' specific surface area (BET) and pore size distribution test were as follows: the parameters such as the specific surface area, pore size, and pore size distribution of the sample are determined by nitrogen isothermal adsorption-desorption method. The first is the preparation of test samples: the test in the experiment requires $20-50 \mathrm{mg}$ of powder sample. Then, we perform thermogravimetric analysis (TGA): the temperature is controlled by the instrument program throughout the process, and the relationship between the quality of the material that changes with time or temperature is measured. It is usually used to detect the composition and thermal stability of materials. In this experiment, TGA was used to detect the components of the sample and the corresponding content of each component [15]. The experimental conditions are air or nitrogen, the flow rate is usually 40 SCCM, the test temperature is gradually increased from $30^{\circ} \mathrm{C}$ to $800^{\circ} \mathrm{C}$, and the sample heating rate is kept at $10^{\circ} \mathrm{C} / \mathrm{min}$, not too fast. The preparation of test samples was as follows: prepare $5 \sim 10 \mathrm{mg}$ powder samples.

\section{Design of Automated Synthesis Equipment}

This study discloses a set of automated synthesis equipment. The design of the equipment mainly includes three parts, namely, system flow, hardware design, and software design [16]. First, open the control software on the computer; you can set the number of various reagents to be added in each reaction position in the control interface. After setting the parameters, the host computer controls the Cartesian robot to move the filling head to the first reaction position to fill the reagent. First, the host computer controls six peristaltic pumps to inject the reagent into the first reaction position and sends it to the lower computer through the serial port: the temperature, heating time, speed (DC motor), and rotation time of the first reaction position. The lower computer receives the temperature, heating time, rotation speed (DC motor), and rotation time of the third reaction position to start temperature control and speed control [17]. Secondly, after the upper computer controls the peristaltic pump to fill the first reagent, it sends a positioning command to the lower computer. After the lower computer receives the positioning command, an IO port generates a trigger signal (low level) [18].

4.1. Hardware Design. SSR solid state relay is used to accurately control the heating power module of the heating mantle, 3144 Hall sensor speed measurement module, based on the L298 N dual H-bridge DC motor drive module to achieve speed control of the magnetic stirring motor, rectangular coordinate robot arm module, and STM32F103ZET6 main controller module [19]. Among them, the PT100 three-wire platinum thermal resistance bridge temperature measurement conversion module is used as the temperature acquisition application of the reaction position, and the measured voltage signal is sent to the main controller ADC port for temperature measurement application. Moreover, the 3144 hall sensor is used as the speed measurement application of the magnetic stirring motor, and the measured pulse signal is sent to the STM32F103ZET6 main controller to realize the speed measurement application. Based on the L298 N dual $\mathrm{H}$-bridge DC motor drive module as the driving application of the magnetic stirring motor, the device uses a Hall sensor to detect the change of the magnetic field. The Hall sensor is based on the Hall effect. For the basis of its work, it is used as a Hall switch in electronic products, such as the flip phone to open the bright screen and close the interest screen [20]. Hall effect: connect the current I to the upper and lower ends of the semiconductor block, and then apply a uniform magnetic field with a magnetic field strength of $B$ in the vertical direction of the semiconductor block. The Hall voltage $U_{\mathrm{H}}$ 
TABLE 1: Other instruments and equipment used in this experiment.

\begin{tabular}{lcc}
\hline Name & Type & Source \\
\hline Vacuum tube furnace & OTF-1200X & Hefei Keying Material Technology Co., Ltd. \\
Vacuum drying oven & DZF-6020 & Shanghai Vichang Technology Co., Ltd. \\
Electric constant temperature blast drying oven & DHG-9070A & Shanghai Vichang Technology Co., Ltd. \\
Electronic balance & BT 25S & Sartorius Scientific Instruments Co., Ltd. \\
Ultrasonic cell crusher & JY92-11N & Ningbo Xianzhi Biotechnology Co., Ltd. \\
Circulating water type multipurpose vacuum pump & SHB-III & Zhengzhou Great Wall Science, Industry and Trade Co., Ltd. \\
\hline
\end{tabular}

will be generated in the direction perpendicular to the current and magnetic field. This effect is the Hall effect, and the relationship is

$$
U_{\mathrm{H}}=K \frac{(B * I)}{D},
$$

where $K$ represents the Hall coefficient, the size of the Hall coefficient is related to the material of the semiconductor wafer, and $D$ represents the thickness of the semiconductor wafer.

4.2. Software Design. The automated synthesis equipment used in this study uses the STM32F103ZET6 ARM chip with Cortex-M3 as the main control chip. The software design of the automatic synthesis equipment control system mainly includes the PT100 three-wire platinum thermosetting resistance bridge temperature measurement conversion module, which is used to measure the voltage signal of the reaction position temperature [21]. The three-wire PT100 platinum thermal resistance bridge temperature conversion module transmits the reaction voltage signal of the temperature measurement to the ADC port through the main controller to realize the temperature measurement program. The PWM output controls the SSR solid state relay to control the electric heating power. The setting procedure for controlling the electric heating temperature is based on 3144 Hall sensors measuring the pulse signal to the main controller, and the TIM implements the speed regulation program [22]. The PWM output controls the DC motor L298 N dual H-bridge driver module to control the application speed of the magnetic stirrer motor and receives the temperature, heating time, speed, rotation time, and step serial port configuration program parameters received from the PC through the serial port.

\section{Automated Synthesis Equipment for Testing the Structure of Carbon Nanomaterial}

In this study, the structure of carbon nanomaterials was tested by automatic synthesis equipment. In this study, the Fourier Transform Infrared Spectrometer (FTIR) in the automatic synthesis system was used for structural testing. The principle is that molecules can selectively absorb infrared rays of certain wavelengths, thereby causing vibrations and rotational energy levels in the molecules. It can also be used to determine the functional groups on the sample surface by measurement [23]. During the experiment, FTIR was used to test Go and characterize the related groups on its surface. The specific test method is as follows: first, the completely dried sample is mixed with potassium tribromide at a determined ratio of three, firstly ground in an agate mortar for about 20 minutes, and then pressed by a tablet press under a pressure of $15 \mathrm{M} \cdot \mathrm{PA}$ for 1 minute to obtain tablets with thickness and density that meet the requirements. Then, the tablet was put in a vacuum oven and dried at $60^{\circ} \mathrm{C}$ for $24 \mathrm{~h}$, and then the sample was tested and identified. The wavenumber range of the test is from $400 \mathrm{~cm}$ to $500 \mathrm{~cm}$. In the study of carbon nanomaterials, the research proves that the structural regularity of carbon nanomaterials and the number of carbon nanolayers can be analyzed by roman spectroscopy [24]. The basic principle of the Raman spectroscopy used is as follows: when monochromatic light illuminates the sample, electrons are excited by absorbing the energy of photons. If the interaction between incident photons and electrons is inelastic scattering, the frequency of the scattered light will be greater or less than the frequency of the incident light, and the scattering process is remanding scattering [25]. The specific test conditions of Raman spectroscopy are as follows: the wavelength of Raman excitation light is $532 \mathrm{~nm}$, and the test wave number is $500 \mathrm{~cm}-2500 \mathrm{~cm}$.

\section{Results and Discussion}

6.1. Performance Analysis of Carbon Nanomaterial Manufacturing System. The research results show that when analyzing the chemical properties of the carbon nanomaterials produced by the carbon nanomaterial manufacturing system, it is found that during the process of program setting, different machine temperatures will have a certain impact on the production of carbon nanomaterials by the machine. The three materials produced by the equipment are labeled UPY, GO, and UGO. The conclusion is that the mass fraction of carbon nanomaterial residues at different temperatures is different. The specific data obtained by the study are shown in Table 2 .

Transmission electron microscopy mainly uses highenergy electron beams for imaging, which are used to project accelerated and concentrated electron beams onto very thin samples. The basic principle of the transmission electron microscope is as follows: once the electrons collide with the atoms in the sample, the direction will change, resulting in a solid angle scattering on the plane, and the angle of the scattering angle is related to the density and thickness of the sample. Samples of different materials have a unique scattering angle, so observation with a transmission electron microscope can form different light and dark images on a 
TABLE 2: Residual mass fraction of carbon nanomaterials at different temperatures.

\begin{tabular}{lccc}
\hline Group & $600^{\circ} \mathrm{C}$ & $700^{\circ} \mathrm{C}$ & $800^{\circ} \mathrm{C}$ \\
\hline UPY & 15.41 & 13.26 & 11.15 \\
GO & 57.83 & 54.44 & 51.89 \\
UGO & 38.99 & 34.06 & 31.24 \\
\hline
\end{tabular}

plane, which is helpful to distinguish the composition and structural differences of the sample. In this study, the characteristics and the low-temperature absorption and desorption ability of the three carbon nanomaterial intermediate layers were mainly analyzed using transmission electron microscopy. The specific results are shown in Figure 1.

It can be seen from the data in Figure 1 that at a temperature of $0.2^{\circ} \mathrm{C}$, the intermediate layers (MPC) of the three materials UPY, GO, and UGO are not easily removed from the carbon nanomaterials, but as the temperature continues to increase, the probability of removing the intermediate layer of the material is increased by about $20 \%-$ $60 \%$ than before. It can be seen that carbon nanomaterials have a certain low-temperature resistance, and the application scenarios are more extensive than ordinary materials, reflecting the advantages of carbon nanomanufacturing equipment. Studies have shown that when X-ray diffraction passes through different crystalline substances, X-rays will generate different degrees of scattering, indicating that different materials have different constituent elements and groups or different structures. The diffraction spectrum of carbon nanoparticles shows different characteristics in terms of number, position, the order of relative intensity, and even the shape of diffraction peaks. The XRD results can be used to study the structure of carbon nanomaterials and the atomic distribution in crystals. Because there are different atomic groups between different layers, the layer spacing of carbon nanomaterials is different. XRD is known to test carbon nanomaterials. After X-ray diffraction analysis, the elemental composition of carbon nanomaterials is shown in Figure 2.

It can be seen from the data in Figure 2 that carbon elements account for the vast majority of carbon nanomaterials, reaching $96.1 \%$, and magnesium elements account for $2.1 \%$. Studies have shown that a certain amount of magnesium elements in carbon nanomaterials can make the structure of carbon nanomaterials. It is more uniform and detailed. In addition, sodium element accounts for $1.1 \%$, titanium element accounts for $0.4 \%$, and phosphorus element accounts for $0.4 \%$. It can be seen that the carbon nanomaterials manufactured by the carbon nanomaterial manufacturing system have certain advantages, reflecting the better performance of the carbon nanomaterial manufacturing system used in this study.

6.2. Control of Automated Synthesis Equipment and Comparison of Carbon Nanomaterial Structures. The control of the automatic equipment is mainly achieved through its function. The main function of the automatic synthesis

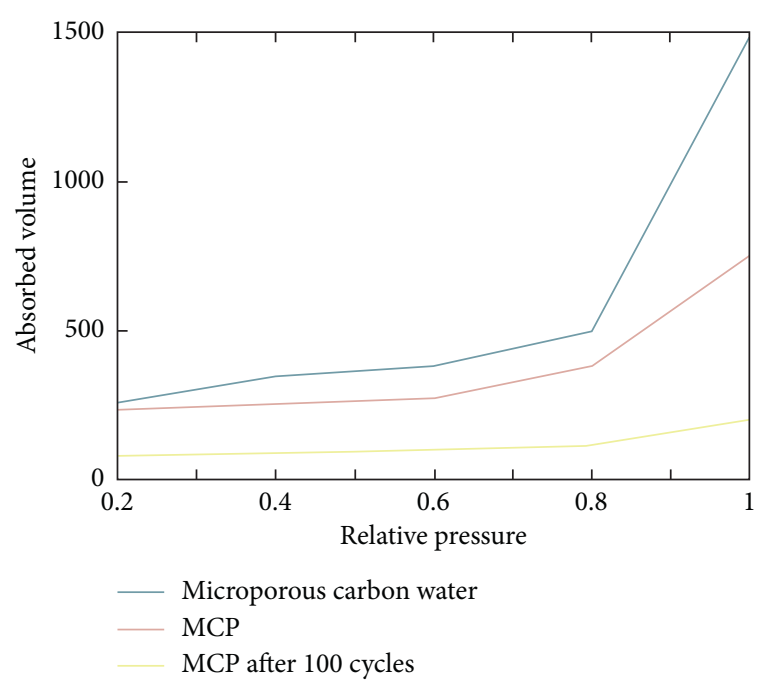

FIGURE 1: Low-temperature adsorption and desorption isotherm diagram of carbon nanomaterial intermediate layer.

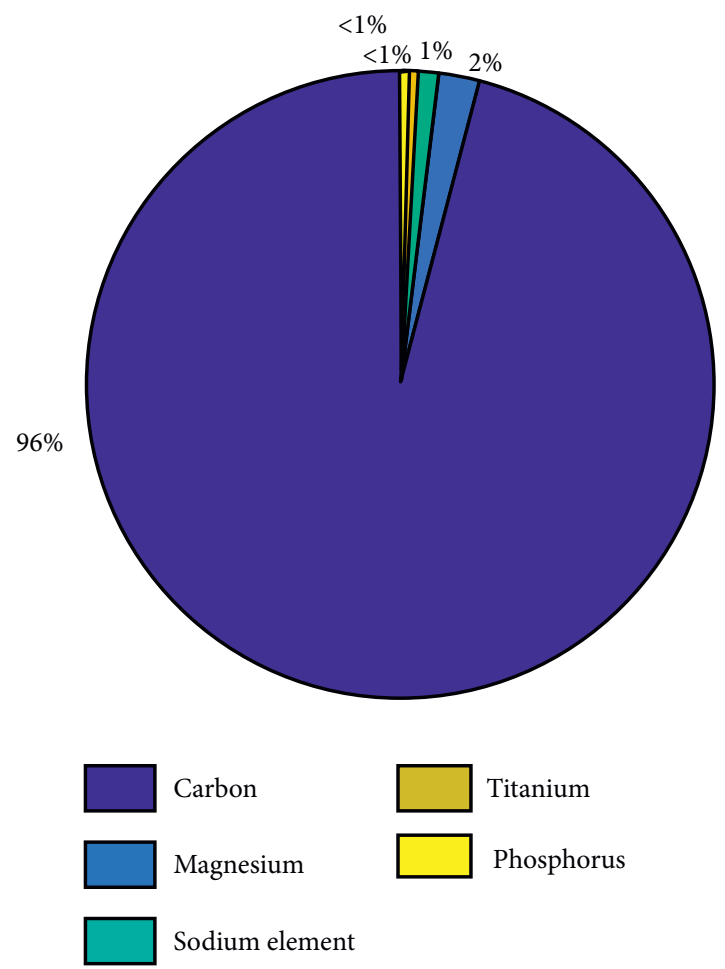

Figure 2: Elemental composition of carbon nanomaterials.

equipment is processed by the main controller through analog-to-digital conversion and then converted to temperature through the algorithm and then through the temperature setpoint, according to the heating temperature difference PWM signal, and then the heating power is changed to achieve temperature control. Then, the Hall sensor module is connected to collect the pulse signal of the STM32 TIM main control terminal. The TM edge set during the period rises to trigger the function, which is used to collect the time and the measured motor speed between the 


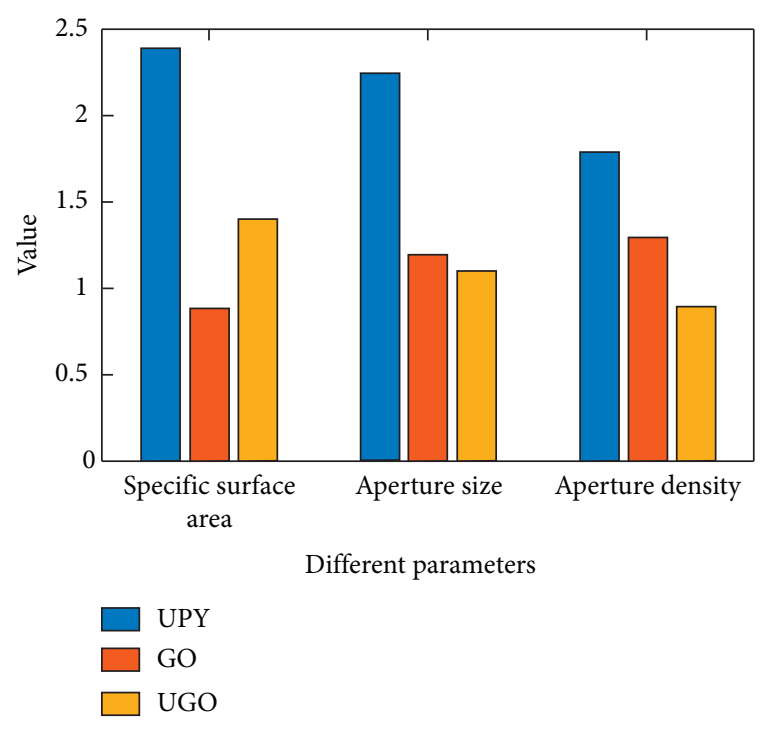

Figure 3: Comparison chart of specific surface area and pore size of three carbon nanomaterials.

two pulse signals. Then, the main controller which is the IO terminal interface of the low-level trigger signal was set to execute the rectangular coordinate robot module, allowing the machine to move smoothly up and down on the machine through the serial port. It should be noted that various parts of the entire system are assigned various tasks to simplify programming difficulty and improve programming efficiency. The biggest competitive advantage of this automated synthesis equipment is the fact that it has extremely high performance, is equipped with the mainstream cortex core, and has strong software support. In this study, the structure of three carbon nanomaterials was tested using automatic synthesis equipment. The specific results are shown in Figure 3.

As can be seen from the data in Figure 3, among the three carbon nanomaterials of UPY, GO, and UGO, UPY has the largest specific surface area, $17 \%$ and $25 \%$ larger than GO and UGO, respectively. Among the three materials, UGO has a pore size of 1.3 and pore density. The smallest is 0.9 . Fourier Transform Infrared Spectroscopy (FTIR) can selectively absorb infrared light of certain wavelengths, thereby causing partial transitions of vibrational energy and rotational energy in the molecule. Studies have shown that the functional groups on the sample surface can be determined by measuring the interferogram and performing flourier changes. FTIR can be used to characterize the groups on the surface of carbon nanomaterials. The specific test method is as follows: mix the completely dried sample with $\mathrm{KBr}$ in a certain ratio, grind it in an agate mortar for $20 \mathrm{~min}$, and then compress it by a tablet press at a pressure of $15 \mathrm{M} \cdot \mathrm{Pa}$ for $1 \mathrm{~min}$ to obtain a tablet. Put the tablet in a vacuum oven, dry at $60^{\circ} \mathrm{C}$ for 24 hours, and then perform testing and characterization analysis. The test wavenumber range is from $500 \mathrm{~nm}$ to $2500 \mathrm{~nm}$. The specific data is shown in Figure 4.

From the data in Figure 4, it can be seen that the three types of carbon nanomaterials UPY, GO, and UGO produced using carbon nanofabrication equipment have the highest frequency of absorbed light when the spectral

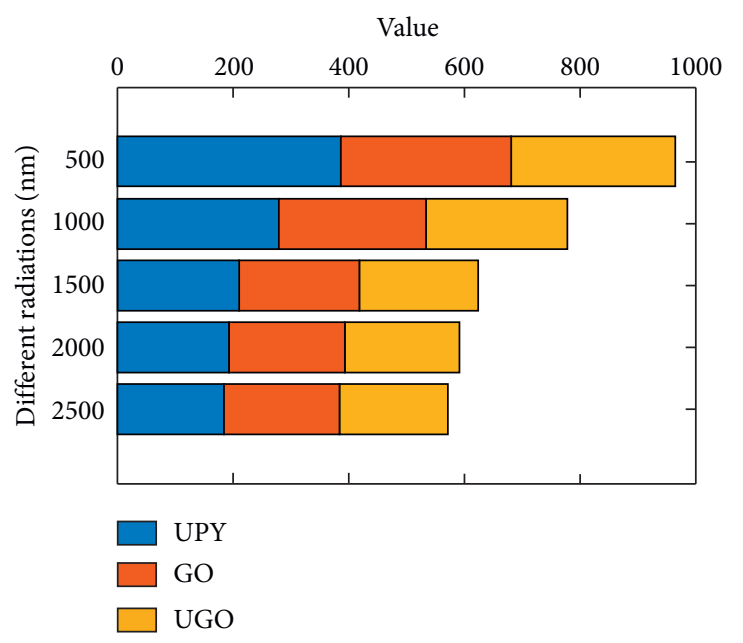

FIgURE 4: Infrared spectrum structure test chart of three carbon nanomaterials.

wavelength is $500 \mathrm{~nm}$, which are 384,296 , and 285 , respectively. The spectrum wavelength is continuously extended, and when the spectrum wavelength is prolonged by 4 times from the beginning, reaching $2500 \mathrm{~nm}$, the frequencies of the three materials UPY, GO, and UGO are reduced by $52 \%, 33 \%$, and $34.7 \%$, respectively. This material is not easy to absorb infrared wavelengths and has the characteristics of being thick and not easy to reflect. In this way, the problems of low efficiency and high cost of carbon nanomaterial production are solved, the production efficiency is increased by $20 \%-35 \%$, and the cost is reduced by $15 \%-30 \%$.

\section{Conclusions}

The research results show that carbon nanotube manufacturing equipment and automated synthesis equipment have great production advantages and can greatly improve the quality and use efficiency of carbon nanomaterials. The three types of carbon nanomaterials produced by carbon nanotube manufacturing equipment, UPY, GO and UGO, are not easy to break. When the wavelength is $500 \mathrm{~nm}$, the three materials have the highest frequency of absorbing light, and with the extension of the spectral wavelength, the frequency of absorbing light of the three materials decreases by $52 \%, 33 \%$, and $34.7 \%$, respectively. The carbon nanomaterial manufacturing system and automated synthesis system studied in this paper solve the problems of high manufacturing cost, low efficiency, and small scale of existing carbon nanomaterials and achieve precision control of automated production. It increases productivity by $20 \%-35 \%$, and the cost is reduced by $15 \%-$ $30 \%$, so they have broad prospects in the fields of science and technology, environmental protection, and intelligent manufacturing.

\section{Data Availability}

The data in this article is available. 


\section{Conflicts of Interest}

The authors declare that they have no conflicts of interest.

\section{Acknowledgments}

This work was supported by the National Natural Science Foundation of China (Grant no. 51305042).

\section{References}

[1] D. M. Fernandes and C. Freire, "Carbon nanomaterialphosphomolybdate composites for oxidative electrocatalysis," Chemelectrochem, vol. 2, no. 2, pp. 269-279, 2015.

[2] S. D. Agata, G. Wroblewski, K. Kielbasinski et al., "Carbon nanomaterials dedicated to heating systems," Circuit World, vol. 41, no. 3, pp. 102-106, 2015.

[3] B.-J. Lee and G.-H. Jeong, "Ferritin-mixed solution plasma system yielding low-dimensional carbon nanomaterials and their application to flexible conductive paper," Current Applied Physics, vol. 15, no. 11, pp. 1506-1511, 2015.

[4] H. Izumi, Y. Okabe, Y. Suda, H. Takikawa, H. Tanoue, and H. Ue, "Manufacturing of electric double-layer capacitors using carbon nanocoils and evaluation of their specific capacitances at a high scan rate," Electronics \& Communications in Japan, vol. 99, no. 5, pp. 3-10, 2016.

[5] Y. Zhang, Z. Kang, X. Yan, and Q. Liao, "Zno nanostructures in enzyme biosensors," Science China Materials, vol. 58, no. 1, pp. 60-76, 2015.

[6] R. Alur and S. Tripakis, "Automatic synthesis of distributed protocols," Acm Sigact News, vol. 48, no. 1, pp. 55-90, 2017.

[7] M. H. A. Khan, H. Thapliyal, and E. Munoz-Coreas, "Automatic synthesis of quaternary quantum circuits," The Journal of Supercomputing, vol. 73, no. 5, pp. 1733-1759, 2017.

[8] V. Y. Osipov, "Automatic synthesis of action programs for intelligent robots," Programming and Computer Software, vol. 42, no. 3, pp. 155-160, 2016.

[9] M. A. Bazhenov, A. V. Shernyukov, M. S. Kupryushkin, and D. V. Pyshnyi, "Study of the staudinger reaction and reveal of key factors affecting the efficacy of automatic synthesis of phosphoryl guanidinic oligonucleotide analogs," Russian Journal of Bioorganic Chemistry, vol. 45, no. 6, pp. 699-708, 2019.

[10] S. Satsangi, C. Patvardhan, and P. K. Kalra, "Automatic synthesis of reversible circuits," International Journal of Computer Applications, vol. 140, no. 11, pp. 31-36, 2016.

[11] Z. Xiao-Jun, L. I. Yun-Gang, and Z. Jin-Ming, "Automatic synthesis and micro imaging study of a serotonin imaging agent," Journal of Isotopes, vol. 28, no. 1, pp. 1-6, 2015.

[12] L. Lessard and S. Lall, "Convexity of decentralized controller synthesis," IEEE Transactions on Automatic Control, vol. 61, no. 10, pp. 3122-3127, 2016.

[13] H. Saggion, "Automatic text simplification," Synthesis Lectures on Human Language Technologies, vol. 10, no. 1, pp. 1137, 2017.

[14] D. Zats, A. P. Iyer, G. Ananthanarayanan, R. Agarwal, R. H. Katz, and Ion Stoica, "Fastlane: making short flows shorter with agile drop notification," Reliability Engineering \& System Safety, vol. 84, no. 1, pp. 19-32, 2016.

[15] A. Janicki, F. Alegre, and N. Evans, "An assessment of automatic speaker verification vulnerabilities to replay spoofing attacks," Security and Communication Networks, vol. 9, no. 15, pp. 3030-3044, 2016.
[16] M. Kimura, "Manufacturing technology using carbon fiber reinforced plastic," Journal of the Japan Society for Precision Engineering, vol. 81, no. 6, pp. 494-497, 2015.

[17] N. A. Agudelo and L. D. Pérez, "Synthesis and characterization of polydimethylsiloxane end-modified polystyrene from poly (styrene-co-vinyltriethoxysilane) copolymers," Materials Research, vol. 19, no. 2, pp. 459-465, 2016.

[18] L. J. D. Oliveira, M. Filgueira, S. C. Cabral, and M. Filgueira, "Study of the TiC coating on powder metallurgy diamonds tool's performance," Materials Research, vol. 18, no. 3, pp. 441-447, 2015.

[19] L. Kojecky, I. Zelinka, and P. Saloun, "Evolutionary synthesis of automatic classification on astroinformatic big data," Parallel Algorithms and Applications, vol. 32, no. 5-6, pp. 429-447, 2017.

[20] L. Thévenoux, P. Langlois, and M. Martel, "Automatic sourceto-source error compensation of floating-point programs: code synthesis to optimize accuracy and time," Journal of Software Maintenance \& Evolution, vol. 29, no. 7, pp. 1-24, 2017.

[21] S. Lee, S. Han, I. Lee, J.-Y. Sim, H.-J. Park, and B. Kim, “Costefficient and automatic large volume data acquisition method for on-chip random process variation measurement," JSTS: Journal of Semiconductor Technology and Science, vol. 15, no. 2, pp. 184-193, 2015.

[22] Y. Li, Q. Liu, R. Tong, and X. Cui, "Shared and service-oriented CNC machining system for intelligent manufacturing process," Chinese Journal of Mechanical Engineering, vol. 28, no. 6, pp. 1100-1108, 2015.

[23] R. Geiger and J. Pahl, "Carbon composite manufacturing in automotive volume production," Lightweight Design Worldwide, vol. 10, no. 4, pp. 46-51, 2017.

[24] P. Hu, "The strategy analysis for Chengdu's industrial carbon emission (manufacturing and environmental management)," Journal of Information \& Management, vol. 31, no. 4, pp. 120-130, 2017.

[25] N. Dugin, T. Zaboronkova, and E. Myasnikov, "Using carbonbased composite materials for manufacturing C-range antenna devices," Latvian Journal of Physics and Technical Sciences, vol. 53, no. 5, pp. 17-23, 2016. 